\title{
Article
}

\section{In Vitro Efficacy of Antibiotic Combinations with Carbapenems and Other Agents against Anaerobic Bacteria}

\author{
Takumi Umemura $^{1}$ (D), Mao Hagihara ${ }^{1,2}$, Takeshi Mori ${ }^{1}$ and Hiroshige Mikamo ${ }^{1, *}$ \\ 1 Department of Clinical Infectious Diseases, Aichi Medical University, Nagakute 480-1195, Japan; \\ umemuratakumi@gmail.com (T.U.); hagimao@aichi-med-u.ac.jp (M.H.); mori99@aichi-med-u.ac.jp (T.M.) \\ 2 Department of Molecular Epidemiology and Biomedical Sciences, Aichi Medical University, \\ Nagakute 480-1195, Japan \\ * Correspondence: mikamo@aichi-med-u.ac.jp; Tel./Fax: +81-561-61-1842
}

check for updates

Citation: Umemura, T.; Hagihara, M.; Mori, T.; Mikamo, H. In Vitro Efficacy of Antibiotic Combinations with Carbapenems and Other Agents against Anaerobic Bacteria. Antibiotics 2022, 11, 292. https:// doi.org/10.3390/antibiotics11030292

Academic Editor: Albert Figueras

Received: 16 January 2022

Accepted: 18 February 2022

Published: 22 February 2022

Publisher's Note: MDPI stays neutral with regard to jurisdictional claims in published maps and institutional affiliations.

Copyright: (c) 2022 by the authors. Licensee MDPI, Basel, Switzerland. This article is an open access article distributed under the terms and conditions of the Creative Commons Attribution (CC BY) license (https:// creativecommons.org/licenses/by/ $4.0 /)$.

\begin{abstract}
We investigated the in vitro efficacy of combinations of carbapenems with clindamycin (CLDM) and minocycline (MINO) against Bacteroides fragilis and Peptostreptococcus species. We selected the carbapenems imipenem, meropenem, panipenem, doripenem, and biapenem. To evaluate the antibiotic efficacy of these combination regimens, the fractional inhibitory concentration index (FICI) was calculated against clinical isolates. Consequently, combination regimens of each carbapenem with CLDM or MINO showed synergistic or additive effects against $83.3-100.0 \%$ and no antagonistic effects against $P$. anaerobius isolates. However, against the B. fragilis group (B. fragilis, B. thetaiotaomicron, and Parabacteroides distasonis), although the combination with other carbapenems and CLDM or MINO did not show remarkable synergistic effects, the combination regimen of IPM with CLDM or MINO indicated mainly additive antibiotic efficacies (FICIs: $>0.5$ to $\leq 1.0$ ) to $B$. fragilis groups. Then, antagonistic effects were admitted in only $5.6 \%$ of $B$. fragilis groups. The effectiveness of antibiotic combination therapy against pathogenic anaerobes has remained unclear. Then, our results can provide new insights to explore the effective combination regimens against multidrug-resistant anaerobic bacteria as empirical and definitive therapies, while this study used only carbapenem susceptible isolates. Hence, further studies are needed to use highly antibiotic-resistant anaerobic isolates to carbapenems.
\end{abstract}

Keywords: carbapenem; clindamycin; minocycline; Bacteroides; Peptostreptococcus; combination therapy; checkerboard assay

\section{Introduction}

The overall number of immunocompromised patients with various disease types has been increasing in recent years with highly advanced medical treatments. This phenomenon resulted in an increase in the number of anaerobic bacteria isolated from patients, especially Bacteroides species [1,2]. Immune insufficiency can cause opportunistic infections, including infections with anaerobes and, then, underlying diseases (e.g., hemodialysis, malignancy and diabetes) are risk factors for anaerobic bacteremia [1]. The presence of anaerobes is known to be associated with a higher rate of mortality, even in polymicrobial infections [3-6]. There have been reports of increased multidrug-resistant Bacteroides fragilis group species, and Peptostreptococcus anaerobius showed the progression of antibiotic resistance worldwide over the last decade [7-11]. Of note, some reference laboratories have reported rates of carbapenem resistance Bacteroides fragilis were up to $7 \%$, whereas resistance to antibiotics has been reported to vary by individual species and geographic regions [12-14]. These bacteria are associated with high mortality rates and are difficult to treat because of their high levels of antibacterial resistance and the lack of effective antibiotic regimens $[15,16]$.

Under these circumstances, clinicians are increasingly using antibiotic combination regimens to treat infected patients. The breakpoint checkerboard assay is one of the methods for selecting optimal antibiotics against multidrug-resistant bacteria [17]. Nakamura 
et al. [18] reported that the clinical cure rate was $83.3 \%$ with antibiotic combination therapy according to the breakpoint checkerboard assay for multidrug-resistant Pseudomonas aeruginosa infections.

Some previous studies have suggested that combination regimens including clindamycin (CLDM) and minocycline (MINO) are effective against certain resistant anaerobes, such as the $B$. fragilis group and P. anaerobius [15,17-20]. Additionally, surveillance studies have demonstrated that carbapenems remain active against the great majority of species within the B. fragilis group [21-24]; thus, carbapenems still play an important role in the development of effective antibiotic combination regimens against multidrug-resistant anaerobes [25].

Nevertheless, few studies have evaluated the antibiotic effect of combination regimens of carbapenems with CLDM or MINO [26,27]. Therefore, this study aimed to investigate the in vitro efficacy of combination regimens of carbapenems with CLDM and MINO against B. fragilis and P. anaerobius.

\section{Results}

\subsection{Antibiotic Susceptibilities of Study Isolates}

Table 1 presents the in vitro susceptibility profiles of carbapenems, CLDM, and MINO against targeted anaerobic bacteria. This study included $18 \mathrm{~B}$. fragilis isolates, $20 \mathrm{~B}$. thetaiotaomicron isolates, 20 Parabacteroides distasonis isolates, and 12 P. anaerobius isolates. For $B$. fragilis, Bacteroides thetaiotaomicron, and P. distasonis isolates, minimum inhibitory concentration (MIC)90 and MIC50 values of imipenem (IPM), meropenem (MEPM), doripenem (DRPM), biapenem (BIPM), and panipenem (PAPM) were $\leq 2$ and $0.75 \mu \mathrm{g} / \mathrm{mL}$, respectively. For P. anaerobius, MIC90 and MIC50 values of IPM, MEPM, DRPM, BIPM, and PAPM were $\leq 1$ and $0.5 \mu \mathrm{g} / \mathrm{mL}$, respectively. For all study isolates, MIC90 and MIC50 of CLDM were $>8$ and $\leq 6 \mu \mathrm{g} / \mathrm{mL}$, respectively. The MIC90 and MIC50 of MINO were 4 (except for B. fragilis, $>8 \mu \mathrm{g} / \mathrm{mL}$ ) and $2 \mu \mathrm{g} / \mathrm{mL}$, respectively.

Table 1. Drug susceptibility testing of anaerobic bacteria.

\begin{tabular}{|c|c|c|c|c|}
\hline & & \multicolumn{3}{|c|}{ MIC ( $\mu \mathrm{g} / \mathrm{mL})$} \\
\hline & & Range & MIC50 & MIC90 \\
\hline B. fragilis & $(n=18)$ & & & \\
\hline IPM & & $0.063-1$ & 0.125 & 0.5 \\
\hline MEPM & & $0.008-1$ & 0.125 & 1 \\
\hline DRPM & & $0.032-1$ & 0.125 & 1 \\
\hline BIPM & & $0.032-2$ & 0.125 & 1 \\
\hline PAPM & & $0.063-2$ & 0.25 & 2 \\
\hline CLDM & & $0.25-8<$ & 0.75 & $8<$ \\
\hline MINO & & $1-8<$ & 2 & $8<$ \\
\hline B. thetaiotaomicron & $(n=20)$ & & & \\
\hline IPM & & $0.25-1$ & 0.5 & 1 \\
\hline MEPM & & $0.032-1$ & 0.125 & 0.25 \\
\hline DRPM & & $0.125-1$ & 0.5 & 0.5 \\
\hline BIPM & & $0.06-0.5$ & 0.25 & 0.5 \\
\hline PAPM & & $0.25-2$ & 0.5 & 2 \\
\hline CLDM & & $1-8<$ & 6 & $8<$ \\
\hline MINO & & $1-4$ & 2 & 4 \\
\hline P. distasonis & $(n=20)$ & & & \\
\hline IPM & & $0.5-2$ & 1 & 2 \\
\hline MEPM & & $0.25-2$ & 0.75 & 2 \\
\hline DRPM & & $0.063-2$ & 0.5 & 2 \\
\hline BIPM & & $0.032-1$ & 0.125 & 0.5 \\
\hline PAPM & & $0.03-2$ & 0.125 & 2 \\
\hline CLDM & & $0.25-8<$ & 4 & $8<$ \\
\hline MINO & & $1-8<$ & 2 & 4 \\
\hline
\end{tabular}


Table 1. Cont.

\begin{tabular}{|c|c|c|c|c|}
\hline & & \multicolumn{3}{|c|}{ MIC $(\mu \mathrm{g} / \mathrm{mL})$} \\
\hline & & Range & MIC50 & MIC90 \\
\hline P. anaerobius & $(n=12)$ & & & \\
\hline IPM & & 0.03-1 & 0.125 & 1 \\
\hline MEPM & & $0.015-1$ & 0.125 & 0.5 \\
\hline DRPM & & $0.06-1$ & 0.5 & 1 \\
\hline BIPM & & $0.03-1$ & 0.125 & 0.5 \\
\hline PAPM & & $0.12-2$ & 0.5 & 1 \\
\hline CLDM & & $0.25-8<$ & 0.5 & $8<$ \\
\hline MINO & & $1-8<$ & 2 & 4 \\
\hline
\end{tabular}

\subsection{Fractional Inhibitory Concentration Index (FICI) of Carbapenems and Clindamycin}

Table 2 demonstrates the FICIs for targeted anaerobic bacteria, and supplementary Figure S1 shows the MIC distribution in combination regimens with carbapenems and CLDM. For $B$. fragilis, each combination regimen displayed synergistic or additive effects in 33.3-38.8\% of the study isolates, whereas MEPM + CLDM and other carbapenems (IPM, DRPM, BIPM, and PAPM) + CLDM showed antagonistic effects to $16.7 \%$ and $5.6 \%$ of the study isolates. For $B$. thetaiotaomicron, each combination regimen indicated synergistic or additive effects in $20.0-60.0 \%$ of the study isolates. Furthermore, IPM + CLDM indicated additive or synergistic effects in more than half of the study isolates $(60.0 \%)$ without showing antagonistic effects. However, the other combination regimens, including MEPM, DRPM, BIPM, and PAPM, indicated antagonistic effects to $5.0-15.0 \%$ of study isolates. For $P$. distasonis, each combination regimen showed additive effects in $25.0-60.0 \%$ of the study isolates. IPM + CLDM displayed additive effects in more than half of the study isolates $(60.0 \%)$ without showing antagonistic effects. However, the other combination regimens, including MEPM, DRPM, BIPM, and PAPM, showed antagonistic effects in 5-15.0\% of the study isolates. For P. anaerobius, each combination regimen indicated synergistic or additive effects in $83.3-100.0 \%$ of the study isolates without indicating any antagonistic effects. IPM + CLDM and MEPM + CLDM indicated synergistic or additive effects in all study isolates $(100.0 \%)$.

Table 2. Fractional inhibitory concentration index (FICI) for anaerobic bacteria in combination with carbapenems plus clindamycin.

\begin{tabular}{ccccc}
\hline & \multicolumn{3}{c}{ FICI } \\
\cline { 2 - 4 } & $\begin{array}{c}\leq \mathbf{0 . 5} \\
\text { Synergistic }\end{array}$ & $\begin{array}{c}>\mathbf{0 . 5} \text { to } \leq \mathbf{1 . 0} \\
\text { Additive }\end{array}$ & $\begin{array}{c}\mathbf{> 1 . 0} \text { to } \leq \mathbf{2 . 0} \\
\text { Indifferent }\end{array}$ & $\begin{array}{c}>\mathbf{2 . 0} \\
\text { Antagonistic }\end{array}$ \\
\hline B. fragilis $(n=18)$ & & & $11(61.1 \%)$ & $1(5.6 \%)$ \\
Imipenem & 0 & $6(33.3 \%)$ & $9(50.0 \%)$ & $3(16.7 \%)$ \\
Meropenem & 0 & $6(33.3 \%)$ & $10(55.6 \%)$ & $1(5.6 \%)$ \\
Doripenem & $3(16.6 \%)$ & $4(22.2 \%)$ & $10(55.6 \%)$ & $1(5.6 \%)$ \\
Biapenem & 0 & $7(38.8 \%)$ & $10(55.6 \%)$ & $1(5.6 \%)$ \\
Panipenem & 0 & $7(38.8 \%)$ & & \\
\hline B. thetaiotaomicron $(n=20)$ & & $8(40.0 \%)$ & 0 \\
Imipenem & $1(5.0 \%)$ & $11(55.0 \%)$ & $15(75.0 \%)$ & $1(5.0 \%)$ \\
Meropenem & 0 & $4(20.0 \%)$ & $11(55.0 \%)$ & $3(15.0 \%)$ \\
Doripenem & 0 & $6(30.0 \%)$ & $8(40.0 \%)$ & $2(10.0 \%)$ \\
Biapenem & 0 & $10(50.0 \%)$ & $13(65.0 \%)$ & $1(5.0 \%)$ \\
Panipenem & 0 & $6(30.0 \%)$ & &
\end{tabular}


Table 2. Cont.

\begin{tabular}{ccccc}
\hline & \multicolumn{3}{c}{ FICI } \\
\cline { 2 - 5 } & $\begin{array}{c}\leq \mathbf{0 . 5} \\
\text { Synergistic }\end{array}$ & $\begin{array}{c}>\mathbf{0 . 5} \text { to } \leq \mathbf{1 . 0} \\
\text { Additive }\end{array}$ & $\begin{array}{c}\mathbf{> 1 . 0} \text { to } \leq \mathbf{2 . 0} \\
\text { Indifferent }\end{array}$ & $\begin{array}{c}>\mathbf{2 . 0} \\
\text { Antagonistic }\end{array}$ \\
\hline $\begin{array}{c}\text { P. distasonis }(n=20) \\
\text { Imipenem }\end{array}$ & 0 & $12(60.0 \%)$ & $8(40.0 \%)$ & 0 \\
Meropenem & 0 & $10(50.0 \%)$ & $9(45.0 \%)$ & $1(5.0 \%)$ \\
Doripenem & 0 & $5(25.0 \%)$ & $12(60.0 \%)$ & $3(15.0 \%)$ \\
Biapenem & 0 & $10(50.0 \%)$ & $8(40.0 \%)$ & $2(10.0 \%)$ \\
Panipenem & 0 & $5(25.0 \%)$ & $14(70.0 \%)$ & $1(5.0 \%)$ \\
\hline P. anaerobius $(n=12)$ & & & & \\
Imipenem & $4(33.3 \%)$ & $8(66.7 \%)$ & 0 & 0 \\
Meropenem & $4(33.3 \%)$ & $8(66.7 \%)$ & 0 & 0 \\
Doripenem & $6(50.0 \%)$ & $4(33.3 \%)$ & $2(16.7 \%)$ & 0 \\
Biapenem & $7(58.4 \%)$ & $4(33.3 \%)$ & $1(8.3 \%)$ & 0 \\
Panipenem & $2(16.7 \%)$ & $8(66.6 \%)$ & $2(16.7 \%)$ & \\
\hline
\end{tabular}

\subsection{FICI of Carbapenems and Minocycline}

Table 3 shows the FICIs for targeted anaerobic bacteria, and Figure S2 shows the MIC distribution in combination regimens with carbapenems and MINO. For B. fragilis, each combination regimen showed synergistic or additive effects in 33.3-72.2\% of the study isolates, whereas IPM + MINO, BIPM + MINO, and PAPM + MINO exhibited antagonistic effects to $5.6 \%, 11.1 \%$, and $5.6 \%$ of the study isolates, respectively. Moreover, IPM + MINO showed the highest synergistic and additive effects in the study isolates $(72.2 \%)$. For B. thetaiotaomicron, each combination regimen showed additive effects to $20.0-75.0 \%$ of the study isolates. No combination regimen showed antagonistic effects, except PAPM + MINO (5.0\%). IPM + CLDM and BIPM + MINO exhibited additive effects in more than half of the study isolates (75.0-60.0\%) without showing antagonistic effects. For P. distasonis, each combination regimen indicated synergistic or additive effects in $30.0-80.0 \%$ of the study isolates, and no combination regimen indicated antagonistic effects. IPM + MINO showed additive effects in more than half of the study isolates $(80.0 \%)$ without showing antagonistic effects. For P. anaerobius, each combination regimen displayed synergistic or additive effects in $91.7-100.0 \%$ of the study isolates without indicating any antagonistic effects. All combination regimens showed synergistic or additive effects in all study isolates (100.0\%), except MEPM + MINO (91.7\%).

Table 3. FICI for anaerobic bacteria in combination with carbapenems plus minocycline.

\begin{tabular}{ccccc}
\hline & \multicolumn{3}{c}{ FICI } \\
\cline { 2 - 5 } & $\begin{array}{c}\leq \mathbf{0 . 5} \\
\text { Synergistic }\end{array}$ & $\begin{array}{c}>\mathbf{0 . 5} \text { to } \leq \mathbf{1 . 0} \\
\text { Additive }\end{array}$ & $\begin{array}{c}\mathbf{> 1 . 0} \text { to } \leq \mathbf{2 . 0} \\
\text { Indifferent }\end{array}$ & $\begin{array}{c}>\mathbf{2 . 0} \\
\text { Antagonistic }\end{array}$ \\
\hline B. fragilis $(n=18)$ & & & \\
Imipenem & $1(5.6 \%)$ & $12(66.6 \%)$ & $4(22.2 \%)$ & $1(5.6 \%)$ \\
Meropenem & 0 & $7(38.9 \%)$ & $11(61.1 \%)$ & 0 \\
Doripenem & 0 & $6(33.3 \%)$ & $12(66.7 \%)$ & 0 \\
Biapenem & 0 & $7(38.9 \%)$ & $9(50.0 \%)$ & $2(11.1 \%)$ \\
Panipenem & 0 & $7(38.9 \%)$ & $10(55.5 \%)$ & $1(5.6 \%)$ \\
\hline B. thetaiotaomicron $(n=20)$ & 0 & $15(75.0 \%)$ & $5(25.0 \%)$ & 0 \\
Imipenem & 0 & $4(20.0 \%)$ & $16(80.0 \%)$ & 0 \\
Meropenem & 0 & $5(25.0 \%)$ & $15(75.0 \%)$ & 0 \\
Doripenem & 0 & $12(60.0 \%)$ & $8(40.0 \%)$ & 0 \\
Biapenem & 0 & $6(30.0 \%)$ & $13(65.0 \%)$ & $1(5.0 \%)$ \\
Panipenem & 0 & & & \\
\hline
\end{tabular}


Table 3. Cont.

\begin{tabular}{ccccc}
\hline & \multicolumn{3}{c}{ FICI } \\
\cline { 2 - 5 } & $\begin{array}{c}\leq \mathbf{0 . 5} \\
\text { Synergistic }\end{array}$ & $\begin{array}{c}>\mathbf{0 . 5} \text { to } \leq \mathbf{1 . 0} \\
\text { Additive }\end{array}$ & $\begin{array}{c}\mathbf{> 1 . 0} \text { to } \leq \mathbf{2 . 0} \\
\text { Indifferent }\end{array}$ & $\begin{array}{c}>\mathbf{2 . 0} \\
\text { Antagonistic }\end{array}$ \\
\hline P. distasonis $(n=20)$ & & & & \\
Imipenem & 0 & $16(80.0 \%)$ & $4(20.0 \%)$ & 0 \\
Meropenem & 0 & $6(30.0 \%)$ & $14(70.0 \%)$ & 0 \\
Doripenem & 0 & $7(35.0 \%)$ & $13(65.0 \%)$ & 0 \\
Biapenem & 0 & $6(30.0 \%)$ & $14(70.0 \%)$ & 0 \\
Panipenem & $1(5.0 \%)$ & $6(30.0 \%)$ & $13(65.0 \%)$ & 0 \\
\hline P. anaerobius $(n=12)$ & & & & \\
Imipenem & $4(33.3 \%)$ & $8(66.7 \%)$ & 0 & 0 \\
Meropenem & $4(33.3 \%)$ & $7(58.3 \%)$ & $1(8.3 \%)$ & 0 \\
Doripenem & $1(8.3 \%)$ & $11(91.7 \%)$ & 0 & 0 \\
Biapenem & 0 & $12(100.0 \%)$ & 0 & 0 \\
Panipenem & $1(8.3 \%)$ & $11(91.7 \%)$ & 0 & \\
\hline
\end{tabular}

\section{Discussion}

Several reports have indicated that multidrug-resistant anaerobic bacteria were detected among clinical isolates [12-14]. This study evaluated the antibiotic activities of combination regimens, including carbapenems (IPM, MEPM, DRPM, BIPM, and PAPM) and CLDM or MINO against anaerobic bacteria using the checkerboard assay. Consequently, these combination regimens showed synergistic or additive antibiotic effects in the majority $(>80.0 \%)$ of $P$. anaerobius isolates. In contrast, the same combination regimens did not show remarkable synergistic effects against some Bacteroides species, such as $B$. fragilis, B. thetaiotaomicron, and P. distasonis (Table 2).

To the best of our knowledge, this is the first study to demonstrate the antibiotic activities of combination regimens, including carbapenems against anaerobic bacteria. CLDM has been commonly used to treat some anaerobic bacterial infections, and MINO has been widely used to treat aerobic and anaerobic bacterial infections, such as odontogenic infections [16]. In clinical practice, IPM is mixed with cilastatin, which works as dehydropeptidase-1 (DHP-1 inhibitor, to prevent IPM degradation by DHP-1 [28]. PAPM was mixed with betamipron (BP), which acts as an organic anion transport inhibitor to reduce renal toxicity [21]. However, in our study, we did not add DHP-1 and BP to evaluate the antibiotic effects of combination regimens, including IPM and PAPM, because these inhibitors have a less antibacterial effect $[29,30]$.

Compared with each single carbapenem regimen, the combination regimens of each carbapenem with CLDM did not enhance antibiotic activities against $B$. fragilis group species in our in vitro study. For most study isolates, FICIs of combination regimens were classified as additive or indifferent effects (Table 2). Kato et al. [22] reported that combination regimens of penicillins and cephalosporins with CLDM showed synergistic effects against $B$. fragilis. However, we observed only a few synergistic effects for the $B$. fragilis group isolates with combination regimens, including each carbapenem and CLDM.

Similar to $B$. fragilis, combination regimens of each carbapenem and CLDM did not cause remarkable MIC changes in B. thetaiotaomicron and P. distasonis (Figure S2B,C). However, FICIs of the combination regimen including IPM + CLDM showed a synergistic or additive effect against the $60 \% \mathrm{~B}$. thetaiotaomicron strains we used. Notably, in a recent report, $8.2 \%$ of $B$. thetaiotaomicron strains were confirmed to be intermediate or resistant to IPM [31], and B. fragilis and B. thetaiotaomicron accounted for a slight share of the observed resistance to IPM and CLDM [32,33]. Hence, a combination regimen of IPM + CLDM can be an option to treat infections caused by multidrug-resistant $B$. thetaiotaomicron.

Furthermore, in combination regimens with each carbapenem and CLDM against P. anaerobius, FICIs of almost all strains showed synergistic or additive effects (Table 3). Brook et al. [34] reported that penicillins combined with CLDM against anaerobic gram- 
positive cocci, including $P$. anaerobius, indicating synergistic or additive effects. Similar to this report, our study results suggest that combination regimens with carbapenems and CLDM have synergistic or additive effects against anaerobic gram-positive cocci (Table 3). Hence, these antibiotic combinations can be an option to treat infections caused by $P$. anaerobius isolates.

In contrast, although the antibiotic effect of combination regimens with $\beta$-lactams and MINO has already been evaluated against aerobic bacteria [35], few studies have evaluated them against anaerobic bacteria. In combination with each carbapenem and MINO, except IPM + MINO against $B$. fragilis, $B$. thetaiotaomicron, and $P$. distasonis, we did not observe remarkable MIC changes between single carbapenem regimens and each combination regimen (Figure S2A-C). In total, 50-70\% of the isolates were classified into an indifferent effect with FICIs. Only IPM + MINO showed synergistic or additive effects against $B$. fragilis group species, but the exact mechanism is unknown.

Compared with single carbapenem regimens, combination regimens with each carbapenem and MINO showed enhanced antibiotic effects on P. anaerobius. The combination regimen resulted in a lower MIC. FICIs were indicated to have synergistic or additive effects in almost all strains. However, the number of isolates used in this study was limited. Hence, although MINO is expected to have a synergistic effect against gram-positive anaerobic isolates, more detailed research using a large number of samples is needed.

Our current study had some limitations. First, the number of clinical isolates evaluated in this in vitro study was small. Related to the limitations, our study results suggested combination regimens of each carbapenem with CLDM or MINO showed not only synergistic or additive effects but also antagonistic effects against the B. fragilis group (5.0-16.7\%). Hence, further research is needed with more isolates to reveal the reason why the same strains showed variate combination effects. Second, our study evaluated the antibiotic activities of some combination regimens only with an in vitro study using checkerboard assay. Although a previous study reported that the results of the checkerboard assay were correlated with clinical response [18], this method does not consider the actual drug transfer to the local infected lesion and the pharmacokinetics of each drug. Additionally, carbapenems change their antibacterial activity under $\mathrm{pH}$ conditions [36]. Hence, various paternal in vivo research, such as a rat model of an abscess, is needed to validate our in vitro results. Third, we admitted the reduction in MICs, for Bacteroides spp., is mostly one dilution, and this may not provide enough reduction to achieve pharmacokinetics/pharmacodynamics targets for more resistant isolates. Finally, the carbapenem concentration evaluated in this study was within the susceptible ranges, and the study isolates we used are limited. Hence, we could not evaluate synergy/antagonism discussion at different MICs tested.

However, the effectiveness of antibiotic combination therapy against pathogenic anaerobes has remained unclear, while some guidelines recommend using combination regimens to treat the infections due to multi-antibiotic resistant aerobic pathogens. We are thinking that one of the reasons is that the data evaluating the effectiveness against anaerobes have been extremely lacking, compared with aerobes. Additionally, reports of multidrug-resistant bacteria have been increasing in anaerobic bacteria. Therefore, our study results would be useful data to explore the preferable antibiotic combination regimens to show enhanced antibiotic activity to clinically important pathogenic anaerobes as preliminary results on in vitro activity of the antibiotic combinations with anti-anaerobic activity. Also, we believe that our results can provide new insights to explore the effective combination regimens against multidrug-resistant anaerobic bacteria as empirical and definitive therapies, while further studies are needed against highly antibiotic-resistant anaerobic isolates to carbapenems.

\section{Materials and Methods}

\subsection{Antibiotics and Checkerboard Production}

We selected IPM, MEPM, PAPM, DRPM, and BIPM as carbapenems and CLDM and MINO. We evaluated the antibiotic efficacy of combination regimens with each carbapenem, 
CLDM, or MINO. Checkerboard plates were purchased from Eiken Chemical Co., Ltd. (Tokyo, Japan). The plates were 96-well microtiter plates and cation-adjusted Brucella broth for susceptibility testing. As shown in Figure 1, each plate comprised of a drug-free medium as a control, a medium containing a single drug to measure MICs, and a medium containing two drugs to evaluate the combined efficacy.

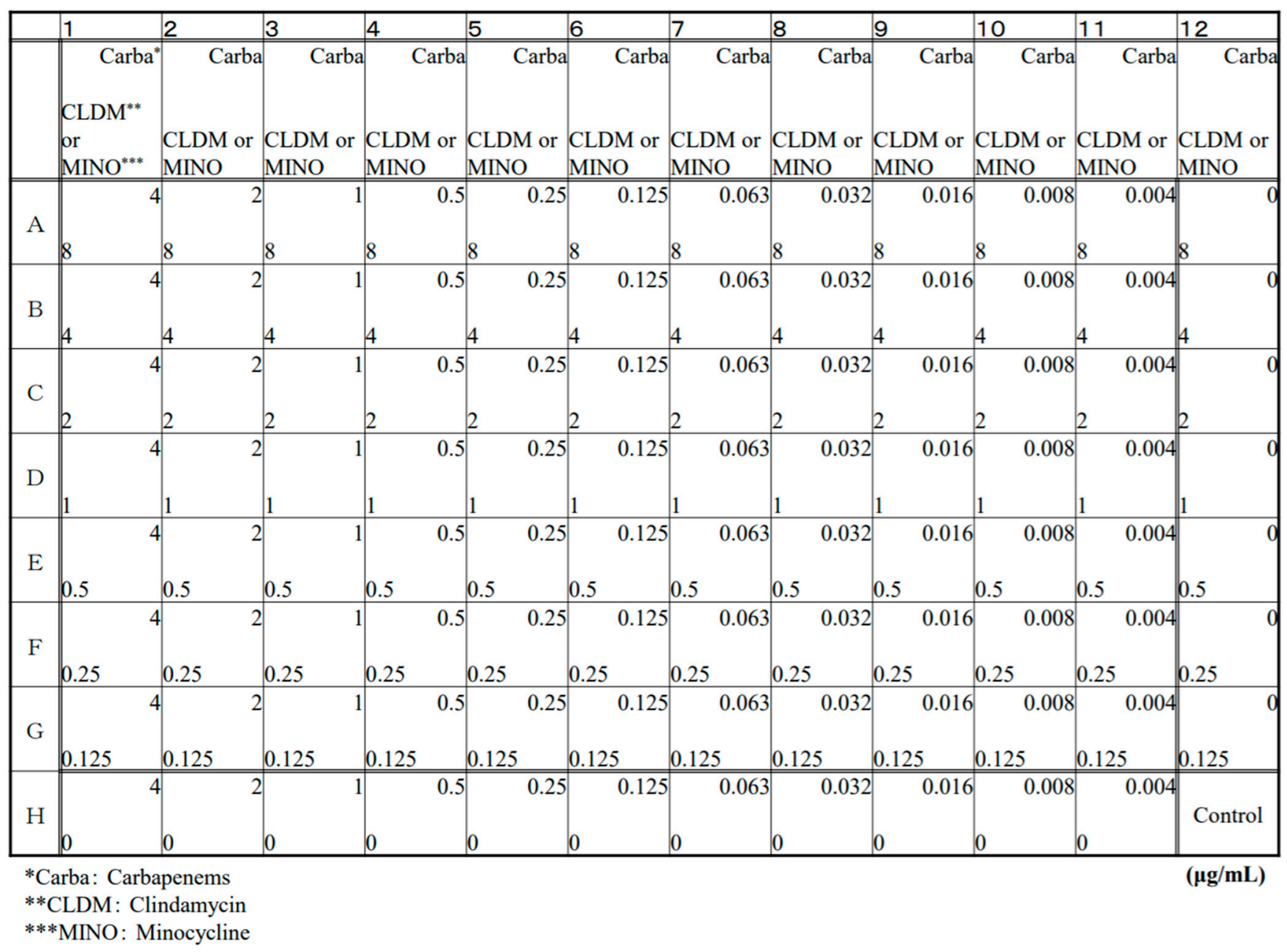

Figure 1. Drug concentration of carbapenems and clindamycin or minocycline by checkerboard assay.

\subsection{Bacterial Strains}

B. fragilis, B. thetaiotaomicron, P. distasonis, and P. anaerobius from clinical isolates were kindly provided by the Gifu University Hospital, Japan. All strains were frozen at $-80{ }^{\circ} \mathrm{C}$ with $15 \%$ skim milk suspension. To evaluate the antibiotic effects of combination regimens with carbapenems and CLDM or MINO, 18 strains of B. fragilis, 20 strains of $B$. thetaiotaomicron, 20 strains of $P$. distasonis, and 12 strains of $P$. anaerobius were tested.

\subsection{Antibiotic Susceptibility Test}

Anaerobic strains were cultured in anaerobic medium gum broth (Nissui Pharmaceutical Inc., Tokyo, Japan) for $24-48 \mathrm{~h}$ at $35 \pm 1{ }^{\circ} \mathrm{C}$. The strains were suspended turbidimetrically at 105 colony forming units (CFU)/well using anaerobic bacterial culture medium (ABCM) broth and ABCM bouillon broth (Eiken Chemical Co., Ltd., Tokyo, Japan). Drug susceptibility tests were demonstrated by broth microdilution method for $46-48 \mathrm{~h}$ at $36 \pm 1^{\circ} \mathrm{C}$, according to the Clinical and Laboratory Standards Institute method [37]. The susceptible MIC break points of the CLSI criteria were as below IPM; $4 \mu \mathrm{g} / \mathrm{mL}$, MEPM; $4 \mu \mathrm{g} / \mathrm{mL}$, DRPM; $2 \mu \mathrm{g} / \mathrm{mL}, \mathrm{CLDM} ; 2 \mu \mathrm{g} / \mathrm{mL}$ and PAPM, BIPM and MINO were unspeci- 
fied). The anaerobic culture apparatus used was an anaerobic box (Hirasawa Works Co., Ltd., Tokyo, Japan).

\subsection{Evaluation of Antibiotic Combination Effect}

To evaluate the antibiotic efficacy of combination regimens with each carbapenem and CLDM or MINO, FICI was calculated as follows: FICI = FIC of carbapenems + FIC of CLDM or MINO, where FIC of carbapenems (with/without CLDM or MINO) was defined as the ratio of the MICs of carbapenems (with/without CLDM or MINO) in combination, and the MIC of carbapenems (with/without CLDM or MINO) alone. The FICI values were interpreted as follows: $\leq 0.5$, synergistic; $>0.5,<2.0$, additive; $>1.0-\leq 2.0$, indifferent; and $>2.0$, antagonistic effects [38].

\section{Conclusions}

This study evaluated the antibiotic activities of combination regimens including carbapenems and CLDM or MINO against anaerobic bacteria using checkerboard assay. Combination regimens of each carbapenem and CLDM or MINO indicated synergistic or additive effects against $P$. anaerobius. Although IPM plus MINO showed mainly additive effects against $B$. fragilis, $B$. thetaiotaomicron, and $P$. distasonis, the other combination regimens including MEPM, DRPM, BIPM, and PAPM with CLDM or MINO showed mainly indifferent effects on the anaerobic isolates in our study. Our results have some potential to provide useful information against future antibiotic-resistant anaerobic strains.

Supplementary Materials: The following are available online at https: / www.mdpi.com/article / 10.3390/antibiotics11030292/s1, Figure S1: Antibacterial activity of carbapenems and clindamycin and their combination against four anaerobes, (A) Antibacterial activity of Carbapenems, Clindamycin and combination against B. fragilis, (B) Antibacterial activity of Carbapenems, Clindamycin and combination against $B$. thetaiotaomicron, (C) Antibacterial activity of Carbapenems, Clindamycin and combination against $P$. distasonis, (D) Antibacterial activity of Carbapenems, Clindamycin and combination against $P$. anaerobius, Figure S2: Antibiotic activity of carbapenems and minocycline and their combination against four anaerobes, (A) Antibacterial activity of Carbapenems, Minocycline and combination against B. fragilis, (B) Antibacterial activity of Carbapenems, Minocycline and combination against B. thetaiotaomicron, (C) Antibacterial activity of Carbapenems, Minocycline and combination against B. distasonis, (D) Antibacterial activity of Carbapenems, Minocycline and combination against $P$. anaerobius.

Author Contributions: T.U., M.H., T.M. and H.M. designed the study and drafted and wrote the manuscript; T.U. performed antimicrobial analysis; All authors have read and agreed to the published version of the manuscript.

Funding: This work received no funding.

Institutional Review Board Statement: Not applicable.

Informed Consent Statement: Not applicable.

Data Availability Statement: The data from the current study can be provided upon reasonable request from the corresponding author.

Acknowledgments: We thank all staff of the Department of Clinical Infectious Diseases, Aichi Medical University Graduate School of Medicine.

Conflicts of Interest: Hiroshige Mikamo received grant support from Shionogi \& Co., Ltd.; Daiichi Sankyo Co., Ltd.; FUJIFILM Toyama Chemical Co., Ltd.; Sumitomo Dainippon Pharma, Co., Ltd.; ASAHI KASEI PHARMA CORPORATION.; and Pfizer Japan Inc., and payment for lectures from MSD K.K.; FUJIFILM Toyama Chemical Co., Ltd.; Miyarisan Pharmaceutical Co., Ltd.; Daiichi Sankyo Co., Ltd.; Pfizer Co., Ltd.; Astellas Pharma Inc., Sumitomo Dainippon Pharma, Co., Ltd.; and Becton, Dickinson and Company. 


\section{References}

1. Ngo, J.T.; Parkins, M.D.; Gregson, D.B.; Pitout, J.D.; Ross, T.; Church, D.L.; Laupland, K.B. Population-based assessment of the inci-dence, risk factors, and outcomes of anaerobic bloodstream infections. Infection 2013, 1, 41-48. [CrossRef]

2. Ali, S.; Dennehy, F.; Donoghue, O.; McNicholas, S. Antimicrobial susceptibility patterns of anaerobic bacteria at an Irish Uni-versity Hospital over a ten-year period (2010-2020). Anaerobe 2021, 7344, 102497.

3. Umemura, T.; Hamada, Y.; Yamagishi, Y.; Suematsu, H.; Mikamo, H. Clinical characteristics associated with mortality of patients with anaerobic bacteremia. Anaerobe 2016, 39, 45-50. [CrossRef]

4. Kim, J.; Lee, Y.; Park, Y.; Kim, M.; Choi, J.Y.; Yong, D.; Jeong, S.H.; Lee, K. Anaerobic Bacteremia: Impact of Inappropriate Therapy on Mortality. Infect. Chemother. 2016, 48, 91-98. [CrossRef]

5. Nguyen, M.H.; Yu, V.L.; Morris, A.J.; McDermott, L.; Wagener, M.W.; Harrell, L.; Snydman, D.R. Antimicrobial Resistance and Clinical Outcome of Bacteroides Bacteremia: Findings of a Multicenter Prospective Observational Trial. Clin. Infect. Dis. 2000, 30, 870-876. [CrossRef]

6. Zahar, J.-R.; Farhat, H.; Chachaty, E.; Meshaka, P.; Antoun, S.; Nitenberg, G. Incidence and clinical significance of anaerobic bacteraemia in cancer patients: A 6-year retrospective study. Clin. Microbiol. Infect. 2005, 11, 724-729. [CrossRef]

7. Copsey-Mawer, S.; Hughes, H.; Scotford, S.; Anderson, B.; Davis, C.; Perry, M.D.; Morris, T.E. UK Bacteroides species surveil-lance survey: Change in antimicrobial resistance over 16 years (2000-2016). Anaerobe 2021, 72, 102447. [CrossRef]

8. Soki, J.; Hedberg, M.; Patrick, S.; Balint, B.; Herczeg, R.; Nagy, I.; Hecht, D.W.; Nagy, E.; Urbán, E. Emergence and evolution of an international cluster of MDR Bacteroides fragilis isolates. J. Antimicrob. Chemother. 2016, 71, 2441-2448. [CrossRef]

9. Nakamura, I.; Aoki, K.; Miura, Y.; Yamaguchi, T.; Matsumoto, T. Fatal sepsis caused by multidrug-resistant Bacteroides fragilis, harboring a cfiA gene and an upstream insertion sequence element, in Japan. Anaerobe 2017, 44, 36-39. [CrossRef]

10. Könönen, E.; Bryk, A.; Niemi, P.; Kanervo-Nordström, A. Antimicrobial Susceptibilities of Peptostreptococcus anaerobius and the Newly Described Peptostreptococcus stomatis Isolated from Various Human Sources. Antimicrob. Agents Chemother. 2007, 51, 2205-2207. [CrossRef]

11. Fujita, K.; Takata, I.; Sugiyama, H.; Suematsu, H.; Yamagishi, Y.; Mikamo, H. Antimicrobial susceptibilities of clinical isolates of the anaerobic bacteria which can cause aspiration pneumonia. Anaerobe 2019, 57, 86-89. [CrossRef]

12. Liu, C.Y.; Huang, Y.T.; Liao, C.H.; Yen, L.C.; Lin, H.Y.; Hsueh, P.R. Increasing trends in antimicrobial resistance among clinically important anaerobes and Bacteroides fragilis isolates causing nosocomial infections: Emerging resistance to carbapenems. Antimicrob Agents Chemother. 2008, 52, 3161-3168. [CrossRef]

13. Jamal, W.; Shahin, M.; Rotimi, V. Surveillance and trends of antimicrobial resistance among clinical isolates of anaerobes in Kuwait hospitals from 2002 to 2007. Anaerobe 2010, 16, 1-5. [CrossRef]

14. Snydman, D.R.; Jacobus, N.V.; McDermott, L.A.; Golan, Y.; Hecht, D.W.; Goldstein, E.J.C.; Harrell, L.J.; Jenkins, S.; Newton, D.; Pierson, C.; et al. Lessons Learned from the Anaerobe Survey: Historical Perspective and Review of the Most Recent Data (2005-2007). Clin. Infect. Dis. 2010, 50, S26-S33. [CrossRef]

15. Ednie, L.M.; Credito, K.L.; Khantipong, M.; Jacobs, M.R.; Appelbaum, P.C. Synergic activity, for anaerobes, of trovafloxacin with clindamycin or metronidazole: Chequerboard and time-kill methods. J. Antimicrob. Chemother. 2000, 45, 633-638. [CrossRef]

16. Inubushi, J.; Liang, K. Update on minocycline in vitro activity against odontogenic bacteria. J. Infect. Chemother. 2020, 26, 1334-1337. [CrossRef]

17. Tateda, K.; Ishii, Y.; Matsumoto, T.; Yamaguchi, K. 'Break-point Checkerboard Plate' for screening of appropriate antibiotic combinations against multidrug-resistant Pseudomonas aeruginosa. Scand. J. Infect. Dis. 2006, 38, 268-272. [CrossRef]

18. Nakamura, I.; Yamaguchi, T.; Tsukimori, A.; Sato, A.; Fukushima, S.; Mizuno, Y.; Matsumoto, T. Effectiveness of antibiotic combination therapy as evaluated by the Break-point Checkerboard Plate method for multidrug-resistant Pseudomonas aeruginosa in clinical use. J. Infect. Chemother. 2014, 4, 266-269. [CrossRef]

19. Whiting, J.L.; Cheng, N.; Chow, A.W. Interactions of ciprofloxacin with clindamycin, metronidazole, cefoxitin, cefotaxime, and mezlocillin against gram-positive and gram-negative anaerobic bacteria. Antimicrob. Agents Chemother. 1987, 31, 1379-1382 [CrossRef]

20. Fass, R.J.; Ruiz, D.E.; Prior, R.B.; Perkins, R.L. In Vitro Activity of Gentamicin and Minocycline Alone and in Combination Against Bacteria Associated with Intra-Abdominal Sepsis. Antimicrob. Agents Chemother. 1976, 10, 34-37. [CrossRef]

21. Takeda, M.; Narikawa, S.; Hosoyamada, M.; Cha, S.H.; Sekine, T.; Endou, H. Characterization of organic anion transport inhibitors using cells stably expressing human organic anion transporters. Eur. J. Pharmacol. 2001, 419, 113-120. [CrossRef]

22. Kato, H.; Kunitomo, W.; Kaori, B.; Kato, N.; Ueno, N. The effect of clindamycin on antibacterial activity of $\beta$-lactam antibiotics against $\beta$-lactamase high-producing Bacteroides fragilis. Prog. Med. 1991, 11, 2647-2652.

23. Zaleznik, D.F.; Zhang, Z.; Onderdonk, A.B.; Kasper, D.L. Effect of Subinhibitory Doses of Clindamycin on the Virulence of Bacteroides fragilis: Role of Lipopolysaccharide. J. Infect. Dis. 1986, 154, 40-46. [CrossRef] [PubMed]

24. Sanders, C.C.; Sanders, W.E., Jr.; Goering, R.V. Influence of Clindamycin on derepression of $\beta$-lactamase in Enterobacter spp. and Pseudomonas aeruginosa. Antimicrob Agents Chemother. 1983, 24, 48-53. [CrossRef] [PubMed]

25. Nagy, E. Anaerobic infections: Update on treatment considerations. Drugs 2010, 70, 841-858. [CrossRef]

26. Heine, H.S.; Shadomy, S.V.; Boyer, A.E.; Chuvala, L.; Riggins, R.; Kesterson, A.; Myrick, J.; Craig, J.; Candela, M.G.; Barr, J.R.; et al. Evaluation of Combination Drug Therapy for Treatment of Antibiotic-Resistant Inhalation Anthrax in a Murine Model. Antimicrob. Agents Chemother. 2017, 61, e00788-e17. [CrossRef] 
27. Beganovic, M.; Daffinee, K.E.; Luther, M.K.; La Plante, K.L. Minocycline Alone and in Combination with Polymyxin B, Meropenem, and Sulbactam against Carbapenem-Susceptible and -Resistant Acinetobacter baumannii in an In Vitro Pharmacodynamic Model. Antimicrob Agents Chemother 2021, 65, e01680-20. [CrossRef]

28. Zhanel, G.G.; Wiebe, R.; Dilay, L.; Thomson, K.; Rubinstein, E.; Hoban, D.J.; Noreddin, A.M.; Karlowsky, J.A. Comparative Review of the Carbapenems. Drugs 2007, 67, 1027-1052. [CrossRef]

29. Shaaban, M.I.; Shaker, M.A.; Mady, F.M. Imipenem/cilastatin encapsulated polymeric nanoparticles for destroying car-bapenemresistant bacterial isolates. J. Nanobiotechnol. 2017, 15, 29. [CrossRef]

30. Goa, K.L.; Noble, S. Panipenem/Betamipron. Drugs 2003, 63, 913-925. [CrossRef]

31. Ho, P.-L.; Yau, C.-Y.; Ho, L.-Y.; Lai, E.L.-Y.; Liu, M.C.-J.; Tse, C.W.-S.; Chow, K.-H. Antimicrobial susceptibility of Bacteroides fragilis group organisms in Hong Kong by the tentative EUCAST disc diffusion method. Anaerobe 2017, 47, 51-56. [CrossRef]

32. Boyanova, L.; Markovska, R.; Mitov, I. Multidrug resistance in anaerobes. Futur. Microbiol. 2019, 14, 1055-1064. [CrossRef]

33. Sadarangani, S.P.; Cunningham, S.A.; Jeraldo, P.R.; Wilson, J.W.; Khare, R.; Patel, R. Metronidazole- and car-bapenem-resistant bacteroides thetaiotaomicron isolated in Rochester, Minnesota. Antimicrob. Agents Chemother. 2015, 59, 4157-4161. [CrossRef] [PubMed]

34. Brook, I.; Walker, R.I. Interaction between penicillin, clindamycin or metronidazole and gentamicin against species of clostridia and anaerobic and facultatively anaerobic Gram-positive cocci. J. Antimicrob. Chemother. 1985, 15, 31-37. [CrossRef] [PubMed]

35. Su, B.A.; Tang, H.J.; Wang, Y.Y.; Liu, Y.C.; Ko, W.C.; Liu, C.Y.; Chuang, Y.C. In vitro antimicrobial effect of cefazolin and cefotaxime combined with minocycline against Vibrio cholerae non-O1 non-O139. J. Microbiol. Immunol. Infect. 2005, 38, 425-429. [PubMed]

36. Matthew, N.L.; Harriet, M.L. Meropenem an updated review of its use in the management of intrrra-abdominal infections. Drugs 2000, 60, 619-641.

37. Clinical and Laboratory Standards Institute. Methods for Dilution Antimicrobial Susceptibility Tests for Bacteria That Grow Aerobically, 9th ed.; Approved Standard; Clinical and Laboratory Standards Institute: Malvern, PA, USA, 2012.

38. Yap, J.K.Y.; Tan, S.Y.Y.; Tang, S.Q.; Thien, V.K.; Chan, E.W.L. Synergistic Antibacterial Activity between 1,4-Naphthoquinone and $\beta$-Lactam Antibiotics against Methicillin-Resistant Staphylococcus aureus. Microb. Drug Resist. 2021, 27, 234-240. [CrossRef] 